TRANSACTIONS OF THE

AMERICAN MATHEMATICAL SOCIETY

Volume 359, Number 10, October 2007, Pages 4711-4727

S 0002-9947(07)04054-8

Article electronically published on May 1, 2007

\title{
MIXED MULTIPLICITIES OF IDEALS VERSUS MIXED VOLUMES OF POLYTOPES
}

\author{
NGO VIET TRUNG AND JUGAL VERMA
}

\begin{abstract}
The main results of this paper interpret mixed volumes of lattice polytopes as mixed multiplicities of ideals and mixed multiplicities of ideals as Samuel's multiplicities. In particular, we can give a purely algebraic proof of Bernstein's theorem which asserts that the number of common zeros of a system of Laurent polynomial equations in the torus is bounded above by the mixed volume of their Newton polytopes.
\end{abstract}

\section{INTRODUCTION}

Let us first recall the definition of mixed volumes. Given two polytopes $P, Q$ in $\mathbb{R}^{n}$ (which need not be different), their Minkowski sum is defined as the polytope

$$
P+Q:=\{a+b \mid a \in P, b \in Q\} .
$$

The $n$-dimensional mixed volume of a collection of $n$ polytopes $Q_{1}, \ldots, Q_{n}$ in $\mathbb{R}^{n}$ is the value

$$
M V_{n}\left(Q_{1}, \ldots, Q_{n}\right):=\sum_{h=1}^{s} \sum_{1 \leq i_{1}<\ldots<i_{h} \leq n}(-1)^{n-h} V_{n}\left(Q_{i_{1}}+\cdots+Q_{i_{h}}\right) .
$$

Here $V_{n}$ denotes the $n$-dimensional Euclidean volume. Mixed volumes play an important role in convex geometry (see $[\mathrm{BF},[\mathrm{Ew})$ and elimination theory (see GKZ, CLO, Stu] .

Our interest in mixed volumes arises from the following result of Bernstein $\mathrm{Be}$ which relates the number of solutions of a system of polynomial equations to the mixed volume of their Newton polytopes (see also [Kh, $[\mathrm{Ku}]$ ).

Bernstein's Theorem. Let $f_{1}, \ldots, f_{n}$ be Laurent polynomials in $\mathbb{C}\left[x_{1}^{ \pm 1}, \ldots, x_{n}^{ \pm 1}\right]$ with finitely many common zeros in the torus $\left(\mathbb{C}^{*}\right)^{n}$. Then the number of common zeros of $f_{1}, \ldots, f_{n}$ in $\left(\mathbb{C}^{*}\right)^{n}$ is bounded above by the mixed volume $M V_{n}\left(Q_{1}, \ldots, Q_{n}\right)$, where $Q_{i}$ denotes the Newton polytope of $f_{i}$. Moreover, this bound is attained for a generic choice of coefficients in $f_{1}, \ldots, f_{n}$.

Bernstein's theorem is a generalization of the classical Bezout's theorem. It is a beautiful example of the interaction between algebra and combinatorics. However, the original proof in $\mathrm{Be}$ has more or less a combinatorial flavor. A geometric proof using intersection theory was given by Teissier [Te3] (see also the expositions

Received by the editors March 1, 2005 and, in revised form, March 30, 2005.

2000 Mathematics Subject Classification. Primary 52B20, 13D40; Secondary 13H15, 05 E99.

Key words and phrases. Mixed volume, mixed multiplicities, multigraded Rees algebra, diagonal algebra, toric rings, Hilbert functions of multigraded algebras.

(C)2007 American Mathematical Society Reverts to public domain 28 years from publication 
Fu2, GKZ]). This paper grew out of our attempt to find an algebraic proof of Bernstein's theorem by using Samuel's multiplicity as it is usually done in a proof of Bezout's theorem. The relationship between toric varieties and multigraded rings used in the geometric proof suggests that mixed multiplicities of ideals may be the link between mixed volume of Newton polytopes of Laurent polynomials and the number of their common zeros. To produce this link we encountered two problems which are of independent interest:

- Can one interpret the number of common zeros of Laurent polynomials in the torus as mixed multiplicity of ideals?

- Does there exist any relationship between mixed multiplicities of ideals and mixed volume of polytopes?

We will solve these problems and we will thereby obtain a proof for Bernstein's theorem which uses mixed multiplicities of ideals in a similar way as Samuel's multiplicity for Bezout's theorem. In fact, the number of common zeros of general polynomials in the torus counted with multiplicities and the mixed volume of their Newton polytopes can be interpreted as the same mixed multiplicity of ideals.

Now we are going to give a brief introduction of mixed multiplicities. Let $J_{1}, \ldots, J_{n}$ be a collection of ideals in a local ring $(A, \mathfrak{m})$ and $I$ an $\mathfrak{m}$-primary ideal. Then the length function

$$
\ell\left(I^{u_{0}} J_{1}^{u_{1}} \cdots J_{n}^{u_{n}} / I^{u_{0}+1} J_{1}^{u_{1}} \cdots J_{n}^{u_{n}}\right)
$$

is a polynomial $P(u)$ for $u_{0}, u_{1}, \ldots, u_{n}$ large enough [Ba], $\mathrm{R} 2$, [Te1]. If we write this polynomial in the form

$$
P(u)=\sum_{\alpha \in \mathbb{N}^{n+1},|\alpha|=r} \frac{1}{\alpha !} e_{\alpha} u^{\alpha}+\{\text { terms of total degree }<r\},
$$

where $r=\operatorname{deg} P(u)$ and $\alpha=\left(\alpha_{0}, \alpha_{1}, \ldots, \alpha_{n}\right)$ of weight

$$
|\alpha|:=\alpha_{0}+\alpha_{1}+\cdots+\alpha_{n}=r, \quad \alpha !:=\alpha_{0} ! \alpha_{1} ! \ldots \alpha_{n} !, \quad u^{\alpha}:=u_{0}^{\alpha_{0}} u_{1}^{\alpha_{1}} \ldots u_{n}^{\alpha_{n}},
$$

then the coefficients $e_{\alpha}$ are non-negative integers. One calls $e_{\alpha}$ the mixed multiplicities of the ideals $I, J_{1}, \ldots, J_{n}$ [Te1]. We will denote $e_{\alpha}$ by $e_{\alpha}\left(I \mid J_{1}, \ldots, J_{n}\right)$. This notion can also be defined for homogeneous ideals in a standard multi-graded algebra over a field. Applications of mixed multiplicities can be found in $[\mathrm{KaV}$, [Ro, [Te1, Te2, [Tr2, Ve1] and Ve2].

If the ideals $J_{1}, \ldots, J_{n}$ are $\mathfrak{m}$-primary ideals, one can interpret $e_{\alpha}\left(I \mid J_{1}, \ldots, J_{n}\right)$ as Samuel's multiplicity of general elements ([Te1], $[\mathrm{R} 2, \overline{\mathrm{Sw}})$ ). However, the techniques used in the $\mathfrak{m}$-primary case are not applicable for non-m-primary ideals. For instance, mixed multiplicities of $\mathfrak{m}$-primary ideals are always positive, whereas they may be zero in the general case. We will develop new techniques to prove the following general result which allows us to test the positivity of mixed multiplicities and to compute them by means of Samuel's multiplicity.

Corollary 1.6. Assume that the local ring $A$ has an infinite residue field. Let $Q$ be an ideal generated by $\alpha_{i}$ general elements in $J_{i}$, for $i=1, \ldots, n$, and $J:=J_{1} \cdots J_{n}$. Then $e_{\alpha}\left(I \mid J_{1}, \ldots, J_{n}\right)>0$ if and only if $\operatorname{dim} A /\left(Q: J^{\infty}\right)=\alpha_{0}+1$. In this case,

$$
e_{\alpha}\left(I \mid J_{1}, \ldots, J_{n}\right)=e\left(I, A /\left(Q: J^{\infty}\right)\right) .
$$


More generally, we can specify a class of concrete ideals $Q$ that can be used to compute $e_{\alpha}\left(I \mid J_{1}, \ldots, J_{n}\right)$ (Theorem 1.4). Such a result was already obtained for two ideals in Tr2]. The novelties here are the use of diagonal subalgebras and the introduction of superficial sequences for a set of ideals which provide a simpler way to study mixed multiplicities. As consequences, we will show that the positivity of $e_{\alpha}\left(I \mid J_{1}, \ldots, J_{n}\right)$ does not depend on the ideal $I$ and is rigid with respect to a certain order of the indices $\alpha$ (Corollary 1.8).

There is already a close relationship between mixed multiplicities of multigraded rings and mixed volumes. First, the multiplicity of a graded toric ring can be expressed in terms of the volume of a convex polytope (which is a consequence of Ehrhart's theory on the number of lattice points in convex polytopes). Second, mixed volume can be defined as a coefficient of the multivariate polynomial representing the volumes of linear combinations of the polytopes (Minkowski formula). Using these facts we find the following interpretation of mixed volumes as mixed multiplicities of ideals.

Corollary 2.5. Let $Q_{1}, \ldots, Q_{n}$ be an arbitrary collection of lattice convex polytopes in $\mathbb{R}^{n}$. Let $A=k\left[x_{0}, x_{1}, \ldots, x_{n}\right]$ and $\mathfrak{m}$ be the maximal graded ideal of $A$. Let $M_{i}$ be any set of monomials of the same degree in $A$ such that $Q_{i}$ is the convex hull of the lattice points of their dehomogenized monomials in $k\left[x_{1}, \ldots, x_{n}\right]$. Let $J_{i}$ be the ideal of A generated by the monomials of $M_{i}$. Then

$$
M V_{n}\left(Q_{1}, \ldots, Q_{n}\right)=e_{(0,1, \ldots, 1)}\left(\mathfrak{m} \mid J_{1}, \ldots, J_{n}\right) .
$$

This interpretation has interesting consequences. For instance, one can deduce properties of mixed volumes from those of mixed multiplicities. Conversely, properties of mixed volumes may predict unknown properties of mixed multiplicities. For instance, well-known inequalities for mixed volumes such as the AlexandroffFenchel inequality (see e.g. $[\mathrm{Kh}, \mathrm{Te} 3$ ) lead us to raise the question whether similar inequalities are valid for mixed multiplicities of ideals (Question 2.7). To give an answer to this question turns out to be a challenging problem.

To prove Bernstein's theorem we first reformulate it for a system of homogeneous polynomial equations. In this case, the number of common zeros of general polynomials $f_{1}, \ldots, f_{n}$ can be seen as Samuel's multiplicity of certain graded algebra. It turn out that this Samuel's multiplicity and the mixed volume of their Newton polytopes are the same mixed multiplicity $e_{(0,1, \ldots, 1)}\left(\mathfrak{m} \mid J_{1}, \ldots, J_{n}\right)$, where $J_{1}, \ldots, J_{n}$ are the ideals generated by the supporting monomials of $f_{1}, \ldots, f_{n}$. By the principle of conservation of number, this implies the bound in Bernstein's theorem for any algebraically closed field (Theorem 3.1).

Finally, we would like to point out that computing mixed volumes is a hard enumerative problem (see e.g. [EC], HS1], HS2]) and that the above relationships between mixed volumes, mixed multiplicities and Samuel multiplicity provide an alternative method for the computation of mixed volumes since many computer algebra programs can compute the Samuel multiplicity or the Hilbert polynomial of multigraded algebras.

This paper is organized as follows. Section 1 will deal with the characterization of mixed multiplicities as Samuel's multiplicities. In Section 2 we will interpret mixed volumes as mixed multiplicities. The algebraic proof of Bernstein's theorem will be given in Section 3 . 


\section{Mixed Multiplicities of ideAls}

We begin with some general observations on Hilbert polynomials of multigraded algebras.

Let $s$ be any non-negative integer. Let $R=\bigoplus_{u \in \mathbb{N}^{s+1}} R_{u}$ be a finitely generated standard $\mathbb{N}^{s+1}$-graded algebra over an Artin local ring $R_{0}$. We say $R$ is standard if it is generated by homogeneous elements of degrees $(0, \ldots, 1, \ldots, 0)$, where 1 occurs only as the $i$ th component, $i=0,1, \ldots, s$. The Hilbert function of $R$ is defined by $H_{R}(u):=\ell\left(R_{u}\right)$, where $\ell$ denotes the length. If we view $u$ as a set of $s+1$ variables $u_{0}, \ldots, u_{s}$, then there exists a polynomial $P_{R}(u)$ and integers $n_{0}, n_{1}, \ldots, n_{s}$ such that $H_{R}(u)=P_{R}(u)$ for $u_{i} \geq n_{i}, i=0,1, \ldots, s$ (abbr. for $u \gg 0$ ) Wa. One calls $P_{R}(u)$ the Hilbert polynomial of $R$. If $P_{R}(u) \neq 0$, we write $P_{R}(u)$ in the form

$$
P_{R}(u)=\sum_{\alpha \in \mathbb{N}^{s+1},|\alpha|=r} \frac{1}{\alpha !} e_{\alpha}(R) u^{\alpha}+\{\text { terms of degree }<r\},
$$

where $r=\operatorname{deg} P_{R}(u)$ and $\alpha=\left(\alpha_{0}, \alpha_{1}, \ldots, \alpha s\right)$ with

$$
|\alpha|:=\alpha_{0}+\alpha_{1}+\cdots+\alpha_{s}=r, \quad \alpha !:=\alpha_{0} ! \alpha_{1} ! \ldots \alpha_{s} !, \quad \text { and } \quad u^{\alpha}:=u_{0}^{\alpha_{0}} u_{1}^{\alpha_{1}} \ldots u_{s}^{\alpha_{s}} .
$$

One calls the coefficients $e_{\alpha}(R)$ the mixed multiplicities of the multigraded algebra $R$. If $s=0$, i.e. $R$ is an $\mathbb{N}$-graded algebra, then $R$ has only one mixed multiplicity. It is the usual multiplicity of $R$, and we will denote it by $e(R)$.

The mixed multiplicities of $R$ can be studied by means of certain $\mathbb{N}$-graded subalgebras. Let $\lambda=\left(\lambda_{0}, \lambda_{1}, \ldots, \lambda_{s}\right)$ be any sequence of non-negative integers. Set

$$
R^{\lambda}:=\bigoplus_{n \geq 0} R_{n \lambda}
$$

Then $R^{\lambda}$ is a finitely generated $\mathbb{N}$-graded algebra over $R_{0}$. One calls $R^{\lambda}$ the $\lambda$ diagonal subalgebra of $R$. This notion plays an important role in the study of embeddings of blowups of projective schemes [CHTV].

Lemma 1.1. Let $r=\operatorname{deg} P_{R}(u) \geq 0$ and let all components of $\lambda$ be positive. Then $\operatorname{dim} R^{\lambda}=r+1$ and

$$
e\left(R^{\lambda}\right)=r ! \sum_{\alpha \in \mathbb{N}^{s+1},|\alpha|=r} \frac{1}{\alpha !} e_{\alpha}(R) \lambda^{\alpha}
$$

Proof. Since all components of $\lambda$ are positive, we have

$P_{R^{\lambda}}(n)=H_{R^{\lambda}}(n)=H_{R}(n \lambda)=\sum_{\alpha \in \mathbb{N}^{s+1},|\alpha|=r} \frac{1}{\alpha !} e_{\alpha}(R) \lambda^{\alpha} n^{r}+\{$ terms of degree $<r\}$

for $n \gg 0$. This implies the conclusion because $\operatorname{dim} R^{\lambda}=\operatorname{deg} P_{R^{\lambda}}(n)+1$.

Let $(A, \mathfrak{m})$ be a local ring (or a standard graded algebra over a field, where $\mathfrak{m}$ is the maximal graded ideal). Let $I$ be an $\mathfrak{m}$-primary ideal and $J_{1}, \ldots, J_{s}$ a sequence of ideals of $A$. One can define the $\mathbb{N}^{s+1}$-graded algebra

$$
R\left(I \mid J_{1}, \ldots, J_{s}\right):=\bigoplus_{\left(u_{0}, u_{1}, \ldots, u_{s}\right) \in \mathbb{N}^{s+1}} I^{u_{0}} J_{1}^{u_{1}} \ldots J_{s}^{u_{s}} / I^{u_{0}+1} J_{1}^{u_{1}} \ldots J_{s}^{u_{s}} .
$$

This algebra can be viewed as the associated graded ring of the Rees algebra $A\left[J_{1} t_{1}, \ldots, J_{s} t_{s}\right]$ with respect to the ideal generated by the elements of $I$. 
For short, set $R=R\left(I \mid J_{1}, \ldots, J_{s}\right)$. Then $R$ is a standard $\mathbb{N}^{s+1}$-graded algebra. Hence it has a Hilbert polynomial $P_{R}(u)$. For any $\alpha \in \mathbb{N}^{s+1}$ with $|\alpha|=\operatorname{deg} P_{R}(u)$ we will set

$$
e_{\alpha}\left(I \mid J_{1}, \ldots, J_{s}\right):=e_{\alpha}(R) .
$$

The mixed multiplicities $e_{\alpha}\left(I \mid J_{1}, \ldots, J_{s}\right)$ were studied first for $\mathfrak{m}$-primary ideals in [Ba], [R1] [R2], Te1] and then for arbitrary ideals in [KaMV], KaV], Tr2], [Vi].

Throughout this section let

$$
\begin{aligned}
J & :=J_{1} \ldots J_{s}, \\
d & :=\operatorname{dim} A /\left(0: J^{\infty}\right),
\end{aligned}
$$

where for any ideal $Q \subset A$ we set $Q: J^{\infty}:=\bigcup_{m>0}\left(Q: J^{m}\right)$. Moreover, for any finitely generated $A$-module $E$ we will denote by $e(\bar{I}, E)$ the Samuel multiplicity of $E$ with respect to $I$.

Theorem 1.2. Let $R=R\left(I \mid J_{1}, \ldots, J_{s}\right)$. Assume that $d=\operatorname{dim} A /\left(0: J^{\infty}\right) \geq 1$. Then

(a) $\operatorname{deg} P_{R}(u)=d-1$,

(b) $e_{(d-1,0, \ldots, 0)}\left(I \mid J_{1}, \ldots, J_{s}\right)=e\left(I, A /\left(0: J^{\infty}\right)\right)$.

Proof. Let $I^{\prime}, J_{1}^{\prime}, \ldots, J_{s}^{\prime}$ be the sequence of ideals generated by $I, J_{1}, \ldots, J_{s}$ in the quotient ring $A /\left(0: J^{\infty}\right)$ and put $R^{\prime}=R\left(I^{\prime} \mid J_{1}^{\prime}, \ldots, J_{s}^{\prime}\right)$. Then

$$
\begin{aligned}
R_{u}^{\prime} & =\left(I^{u_{0}} J_{1}^{u_{1}} \ldots J_{s}^{u_{s}}+\left(0: J^{\infty}\right)\right) /\left(I^{u_{0}+1} J_{1}^{u_{1}} \ldots J_{s}^{u_{s}}+\left(0: J^{\infty}\right)\right) \\
& =I^{u_{0}} J_{1}^{u_{1}} \ldots J_{s}^{u_{s}} /\left(I^{u_{0}+1} J_{1}^{u_{1}} \ldots J_{s}^{u_{s}}+I^{u_{0}} J_{1}^{u_{1}} \ldots J_{s}^{u_{s}} \cap\left(0: J^{\infty}\right)\right) .
\end{aligned}
$$

Since $I^{u_{0}} J_{1}^{u_{1}} \ldots J_{s}^{u_{s}} \cap\left(0: J^{\infty}\right)=0$ for $u \gg 0$, we get $R_{u}=R_{u}^{\prime}$ for $u \gg 0$. Hence

$$
P_{R}(u)=P_{R^{\prime}}(u) \text {. }
$$

So we may replace $A$ by $A /\left(0: J^{\infty}\right)$. If we do so, we may assume that $0: J^{\infty}=0$ and $d=\operatorname{dim} A \geq 1$. Then ht $J \geq 1$. For $\lambda=(1, \ldots, 1)$ we have

$$
R^{\lambda}=\bigoplus_{n \geq 0} I^{n} J^{n} / I^{n+1} J^{n} \cong A[I J t] /(I),
$$

where $A[I J t]$ is the Rees algebra of the ideal $I J$. Since $\operatorname{ht}(I J) \geq 1$, we have $\operatorname{dim} A[I J t]=d+1$ Va, Corollary 1.6]. Hence $\operatorname{dim} R^{\lambda} \leq d$. By Lemma 1.1, this implies $\operatorname{deg} P_{R}(u) \leq d-1$.

On the other hand, $\operatorname{dim} A / J^{m}<d$ for any $m \geq 1$. Therefore,

$$
e(I, A)=e\left(I, J^{m}\right)=\lim _{n \rightarrow \infty} \frac{\ell\left(I^{n} J^{m} / I^{n+1} J^{m}\right)}{n^{d-1} /(d-1) !}=\lim _{n \rightarrow \infty} \frac{P_{R}(n, m, \ldots, m)}{n^{d-1} /(d-1) !}
$$

for $m \gg 0$. Since $e(I, A)>0$, this implies $\operatorname{dim} P_{R}(u) \geq d-1$. So we can conclude that $\operatorname{deg} P_{R}(u)=d-1$ and that $e_{(d-1,0, \ldots, 0)}(R)=e(I, A)$.

The computation of mixed multiplicities can be passed to the case $e_{(d-1,0, \ldots, 0)}(R)$. For this we shall need the following notation.

Given a standard $\mathbb{Z}^{s+1}$-graded algebra $S$, we will denote by $S_{+}$the ideal of $S$ generated by the homogeneous elements of degrees with positive components. A sequence of homogeneous elements $z_{1}, \ldots, z_{m}$ in $S$ is called filter-regular if

$$
\left[\left(z_{1}, \ldots, z_{i-1}\right): z_{i}\right]_{u}=\left(z_{1}, \ldots, z_{i-1}\right)_{u}
$$

for $u \gg 0, i=1, \ldots, m$. It is easy to see that this is equivalent to the condition $z_{i} \notin P$ for any associated prime $P \nsupseteq S_{+}$of $S /\left(z_{1}, \ldots, z_{i-1}\right)$. 
Remark. Filter-regular sequences have their origin in the theory of Buchsbaum rings [SV, Appendix]. It can be shown that if $S$ is a standard graded algebra over a field, then $\operatorname{Proj}(S)$ is an equidimensional Cohen-Macaulay scheme if and only if every homogeneous system of parameters of $S$ is filter-regular.

We will now work in the $\mathbb{Z}^{s+1}$-graded algebra

$$
S:=\bigoplus_{u \in \mathbb{Z}^{s+1}} I^{u_{0}} J_{1}^{u_{1}} \ldots J_{s}^{u_{s}} / I^{u_{0}+1} J_{1}^{u_{1}+1} \ldots J_{s}^{u_{s}+1} .
$$

Let $\varepsilon_{1}, \ldots, \varepsilon_{m}$ be any non-decreasing sequence of indices with $1 \leq \varepsilon_{i} \leq s$. Let $x_{1}, \ldots, x_{m}$ be a sequence of elements of $A$ with $x_{i} \in J_{\varepsilon_{i}}, i=1, \ldots, m$. We denote by $x_{i}^{*}$ the residue class of $x_{i}$ in $J_{\varepsilon_{i}} / I J_{1} \ldots J_{\varepsilon_{i}-1} J_{\varepsilon_{i}}^{2} J_{\varepsilon_{i}+1} \ldots J_{s}$. We call $x_{1}, \ldots, x_{m}$ an $\left(\varepsilon_{1}, \ldots, \varepsilon_{m}\right)$-superficial sequence for the ideals $J_{1}, \ldots, J_{s}$ (with respect to $I$ ) if $x_{1}^{*}, \ldots, x_{m}^{*}$ is a filter-regular sequence in $S$.

The above notion can be considered as a generalization of the classical notion of a superficial element of an ideal, which plays an important role in the theory of multiplicity. Recall that an element $x$ is called superficial with respect to an ideal $\mathfrak{a}$ if there is an integer $c$ such that

$$
\left(\mathfrak{a}^{n}: x\right) \cap \mathfrak{a}^{c}=\mathfrak{a}^{n-1}
$$

for $n \gg 0$. A sequence of elements $x_{1}, \ldots, x_{m} \in \mathfrak{a}$ is called a superficial sequence of $\mathfrak{a}$ if the residue class of $x_{i}$ in $A /\left(x_{1}, \ldots, x_{i-1}\right)$ is a superficial element of the ideal $\mathfrak{a} /\left(x_{1}, \ldots, x_{i-1}\right), i=1, \ldots, m$. It is known that this is equivalent to the condition that the initial forms of $x_{1}, \ldots, x_{m}$ in $\mathfrak{a} / \mathfrak{a}^{2}$ form a filter-regular sequence in the associated graded ring $\bigoplus_{n \geq 0} \mathfrak{a}^{n} / \mathfrak{a}^{n+1}$ (see e.g. [Tr1, Lemma 6.2]).

We may use superficial sequences to reduce the dimension of the base ring.

Lemma 1.3. Let $Q$ be an ideal of $A$ generated by an $\left(\varepsilon_{1}, \ldots, \varepsilon_{m}\right)$-superficial sequence of $J_{1}, \ldots, J_{s}$. Let $\bar{I}, \bar{J}_{1}, \ldots, \bar{J}_{s}$ be the sequence of ideals generated by $I, J_{1}, \ldots, J_{s}$ in the quotient ring $A / Q$ and put $\bar{R}=R\left(\bar{I} \mid \bar{J}_{1}, \ldots, \bar{J}_{s}\right)$. Let $\alpha_{j}$ be the number of the indices $i$ such that $\varepsilon_{i}=j, j=1, \ldots, s$. Let $\Delta^{\left(0, \alpha_{1}, \ldots, \alpha_{s}\right)} P_{R}(u)$ denote the $\left(0, \alpha_{1}, \ldots, \alpha_{s}\right)$ difference of the polynomial $P_{R}(u)$. Then

$$
P_{\bar{R}}(u)=\Delta^{\left(0, \alpha_{1}, \ldots, \alpha_{s}\right)} P_{R}(u) .
$$

Proof. If $m=1$, we may assume that $\left(\alpha_{1}, \ldots, \alpha_{s}\right)=(1,0, \ldots, 0)$. Then $Q=(x)$, where $x \in J_{1}$ such that $\left(0: x^{*}\right)_{u}=0$ for $u \gg 0$. This means

$$
\left(I^{u_{0}+1} J_{1}^{u_{1}+2} J_{2}^{u_{2}+1} \ldots J_{s}^{u_{s}+1}: x\right) \cap I^{u_{0}} J_{1}^{u_{1}} \ldots J_{s}^{u_{s}}=I^{u_{0}+1} J_{1}^{u_{1}+1} \ldots J_{s}^{u_{s}+1} .
$$

As a consequence we get

$$
\left(I^{u_{0}+1} J_{1}^{u_{1}+2} J_{2}^{u_{2}+1} \ldots J_{s}^{u_{s}+1}: x\right) \cap I^{u_{0}} J_{1}^{u_{1}+1} \ldots J_{s}^{u_{s}+1}=I^{u_{0}+1} J_{1}^{u_{1}+1} \ldots J_{s}^{u_{s}+1}
$$

for $u \gg 0$. Consider $R$ as a quotient ring of $S$. The above formula shows that $\left(0_{R}: x^{*}\right)_{u}=0$ for $u \gg 0$. Hence $P_{R /\left(0_{R}: x^{*}\right)}(u)=P_{R}(u)$. Now, from the exact sequence

$$
0 \longrightarrow R /\left(0:_{R} x^{*}\right) \stackrel{x^{*}}{\longrightarrow} R \longrightarrow R /\left(x^{*}\right) \longrightarrow 0
$$

we can deduce that

$$
P_{R /\left(x^{*}\right)}(u)=\Delta^{(0,1,0, \ldots, 0)} P_{R}(u) .
$$

On the other hand, (1) implies

$$
I^{u_{0}+1} J_{1}^{u_{1}+2} \ldots J_{s}^{u_{s}+1} \cap x I^{v_{0}} J_{1}^{v_{1}} \ldots J_{s}^{v_{s}}=x I^{u_{0}+1} J_{1}^{u_{1}+1} \ldots J_{s}^{u_{s}+1}
$$


for $\left(u_{0}, u_{1}, \ldots, u_{s}\right) \gg 0$ and $v_{i}=u_{i}, u_{i}+1, i=0,1, \ldots, s$. Using this formula we can easily show that

$$
I^{u_{0}} J_{1}^{u_{1}} \ldots J_{s}^{u_{s}} \cap x I^{v_{0}} J_{1}^{v_{1}} \ldots J_{s}^{v_{s}}=x I^{\max \left\{u_{0}, v_{0}\right\}} J_{1}^{\max \left\{u_{1}-1, v_{1}\right\}} \ldots J_{s}^{\max \left\{u_{s}, v_{s}\right\}}
$$

for $u \gg 0, v \gg 0$. By the Artin-Rees lemma, there exists $\left(c_{0}, c_{1}, \ldots, c_{s}\right) \in \mathbb{N}^{s+1}$ with $c_{1}>0$ such that

$$
I^{u_{0}} J_{1}^{u_{1}} \ldots J_{s}^{u_{s}} \cap(x) \subseteq x I^{u_{0}-c_{0}} J_{1}^{u_{1}-c_{1}} \ldots J_{s}^{u_{s}-c_{s}}
$$

for $u_{i} \geq c_{i}, i=0,1, \ldots, s$. Therefore,

$$
\begin{aligned}
I^{u_{0}} J_{1}^{u_{1}} \ldots J_{s}^{u_{s}} \cap(x) & =I^{u_{0}} J_{1}^{u_{1}} \ldots J_{s}^{u_{s}} \cap x I^{u_{0}-c_{0}} J_{1}^{u_{1}-c_{1}} \ldots J_{s}^{u_{s}-c_{s}} \\
& =x I^{u_{0}} J_{1}^{u_{1}-1} J_{2}^{u_{2}} \ldots J_{s}^{u_{s}}
\end{aligned}
$$

for $u \gg 0$. This implies

$$
\begin{aligned}
\bar{R}_{u} & =\left(I^{u_{0}} J_{1}^{u_{1}} \ldots J_{s}^{u_{s}}, x\right) /\left(I^{u_{0}+1} J_{1}^{u_{1}} \ldots J_{s}^{u_{s}}, x\right) \\
& =I^{u_{0}} J_{1}^{u_{1}} \ldots J_{s}^{u_{s}} /\left(I^{u_{0}+1} J_{1}^{u_{1}} \ldots J_{s}^{u_{s}}+I^{u_{0}} J_{1}^{u_{1}} \ldots J_{s}^{u_{s}} \cap(x)\right) \\
& =I^{u_{0}} J_{1}^{u_{1}} \ldots J_{s}^{u_{s}} /\left(I^{u_{0}+1} J_{1}^{u_{1}} \ldots J_{s}^{u_{s}}+x I^{u_{0}} J_{1}^{u_{1}-1} J_{2}^{u_{2}} \ldots J_{s}^{u_{s}}\right) \\
& =\left(R /\left(x^{*}\right)\right)_{u} .
\end{aligned}
$$

Thus, $P_{\bar{R}}(u)=P_{R /\left(x^{*}\right)}(u)$. Combining this with $(2)$ we get $P_{\bar{R}}(u)=\Delta^{(0, . ., 1, . ., 0)} P_{R}(u)$ which proves the case $m=1$.

If $m>1$, we may assume that $\alpha_{1}>0$. Then $x_{1} \in J_{1}$. Let $I^{*}, J_{1}^{*}, \ldots, J_{s}^{*}$ denote the sequence of the ideals generated by $I, J_{1}, \ldots, J_{s}$ in the quotient ring $A /\left(x_{1}\right)$. Put $R^{*}=R\left(I^{*} \mid J_{1}^{*}, \ldots, J_{s}^{*}\right)$. As shown above, we have

$$
P_{R^{*}}(u)=\Delta^{(0,1,0, \ldots, 0)} P_{R}(u) .
$$

Let $S^{*}:=\bigoplus_{u \in \mathbb{Z}^{s+1}}\left(I^{*}\right)^{u_{0}}\left(J_{1}^{*}\right)^{u_{1}} \ldots\left(J_{s}^{*}\right)^{u_{s}} /\left(I^{*}\right)^{u_{0}+1}\left(J_{1}^{*}\right)^{u_{1}+1} \ldots\left(J_{s}^{*}\right)^{u_{s}+1}$. For $u \gg 0$ we have

$$
\begin{aligned}
{\left[S /\left(x_{1}^{*}\right)\right]_{u} } & =I^{u_{0}} J_{1}^{u_{1}} \ldots J_{s}^{u_{s}} /\left(I^{u_{0}+1} J_{1}^{u_{1}+1} \ldots J_{s}^{u_{s}+1}+x_{1} I^{u_{0}} J_{1}^{u_{1}-1} J_{2}^{u_{2}} \ldots J_{s}^{u_{s}}\right) \\
& =I^{u_{0}} J_{1}^{u_{1}} \ldots J_{s}^{u_{s}} /\left(I^{u_{0}+1} J_{1}^{u_{1}+1} \ldots J_{s}^{u_{s}+1}+\left(x_{1}\right) \cap I^{u_{0}} J_{1}^{u_{1}} \ldots J_{s}^{u_{s}}\right) \\
& =\left(I^{u_{0}} J_{1}^{u_{1}} \ldots J_{s}^{u_{s}}, x_{1}\right) /\left(I^{u_{0}+1} J_{1}^{u_{1}+1} \ldots J_{s}^{u_{s}+1}, x_{1}\right) \\
& =S_{u}^{*}
\end{aligned}
$$

Since $\left[\left(x_{1}^{*}, \ldots, x_{i-1}^{*}\right): x_{i}\right]_{u}=\left(x_{1}^{*}, \ldots, x_{i-1}^{*}\right)_{u}$ for $u \gg 0, i=2, \ldots, m$, we also have

$$
\left[\left(x_{2}^{*}, \ldots, x_{i-1}^{*}\right) S^{*}: x_{i}\right]_{u}=\left(x_{2}^{*}, \ldots, x_{i-1}^{*}\right) S_{u}^{*}
$$

for $u \gg 0, i=2, \ldots, m$. Therefore, $x_{2}^{*}, \ldots, x_{m}^{*}$ is an $\left(\varepsilon_{1}-1, \varepsilon_{2}, \ldots, \varepsilon_{s}\right)$-superficial sequence of the ideals $J_{1}^{*}, \ldots, J_{s}^{*}$. Now, we may use induction on $m$ to assume that

$$
P_{\bar{R}}(u)=\Delta^{\left(0, \alpha_{1}-1, \alpha 2, \ldots, \alpha s\right)} P_{R^{*}}(u) .
$$

Combining this with (3) we get $P_{\bar{R}}(u)=\Delta^{\left(0, \alpha_{1}, \alpha 2, \ldots, \alpha s\right)} P_{R}(u)$.

Using Theorem 1.2 and Lemma 1.3 we obtain the following criterion for the positivity of mixed multiplicities.

Theorem 1.4. Let $\alpha=\left(\alpha_{0}, \alpha_{1}, \ldots, \alpha s\right)$ be any sequence of non-negative integers with $|\alpha|=d-1$. Let $Q$ be any ideal generated by an $\left(\alpha_{1}, \ldots, \alpha_{s}\right)$-superficial sequence 
of the ideals $I, J_{1}, \ldots, J_{s}$. Then $e_{\alpha}\left(I \mid J_{1}, \ldots, J_{s}\right)>0$ if and only if $\operatorname{dim} A /\left(Q: J^{\infty}\right)=$ $\alpha_{0}+1$. In this case,

$$
e_{\alpha}\left(I \mid J_{1}, \ldots, J_{s}\right)=e\left(I, A /\left(Q: J^{\infty}\right)\right) .
$$

Proof. If $\alpha=(d-1,0, \ldots, 0)$, the conclusion follows from Theorem 1.2, If $\alpha \neq$ $(d-1,0, \ldots, 0)$, then $d \geq 2$. Let $\bar{R}, \bar{I}, \bar{J}_{1}, \ldots, \bar{J}_{s}$ be as in Lemma 1.3. Then $\operatorname{deg} P_{\bar{R}}(u) \leq$ $d-1-m=\alpha_{0}$ where $m=\alpha_{1}+\cdots+\alpha_{s}$. Write

$$
P_{\bar{R}}(u)=\sum_{\beta \in \mathbb{N}^{s+1},|\beta|=\alpha_{0}} \frac{e_{\beta}\left(\bar{I} \mid \bar{J}_{1}, \ldots, \bar{J}_{s}\right)}{\beta !} u^{\beta}+\left\{\text { terms of degree }<\alpha_{0}\right\} .
$$

Then

$$
e_{\left(\alpha_{0}, \alpha_{1}, \ldots, \alpha_{s}\right)}\left(I \mid J_{1}, \ldots, J_{s}\right)=e_{\left(\alpha_{0}, 0, \ldots, 0\right)}\left(\bar{I} \mid \bar{J}_{1}, \ldots, \bar{J}_{s}\right) .
$$

If $e_{\alpha}\left(I \mid J_{1}, \ldots, J_{s}\right)>0$, then $e_{\left(\alpha_{0}, 0, \ldots, 0\right)}\left(\bar{I} \mid \bar{J}_{1}, \ldots, \bar{J}_{s}\right)>0$. Therefore, $\operatorname{deg} P_{\bar{R}}(u)=$ $\alpha_{0}$. By Theorem 1.2(a), this implies $\operatorname{dim} A /\left(Q: J^{\infty}\right)=\alpha_{0}+1$.

Conversely, if $\operatorname{dim} A /\left(Q: J^{\infty}\right)=\alpha_{0}+1$ and if we put $\bar{J}=\bar{J}_{1} \ldots \bar{J}_{s}$, then

$$
e_{\left(\alpha_{0}, 0, \ldots, 0\right)}\left(\bar{I} \mid \bar{J}_{1}, \ldots, \bar{J}_{s}\right)=e\left(\bar{I}, \bar{A} /\left(0: \bar{J}^{\infty}\right)\right)=e\left(I, A /\left(Q: J^{\infty}\right)\right)
$$

by Theorem 1.2(b). Since the Samuel multiplicity is always positive, this implies $e_{\left(\alpha_{0}, 0, \ldots, 0\right)}\left(\bar{I} \mid \bar{J}_{1}, \ldots, \bar{J}_{s}\right)>0$. So we can conclude that $e_{\alpha}\left(I \mid J_{1}, \ldots, J_{s}\right)>0$ if and only if $\operatorname{dim} A /\left(Q: J^{\infty}\right)=\alpha_{0}+1$.

Let $k$ be the residue field of $A$. Using the prime avoidance characterization of a superficial element we can easily see that superficial sequences exist if $k$ is infinite. In fact, general elements of $J_{1}, \ldots, J_{s}$ always form a superficial sequence. Recall that a property holds for a general element $x$ of an ideal $Q=\left(y_{1}, \ldots, y_{m}\right)$ if there exists a non-empty Zariski-open subset $U \subseteq k^{m}$ such that whenever $x=\sum_{j=1}^{m} c_{j} x_{j}$ and the image of $\left(c_{1}, \ldots, c_{m}\right)$ in $k^{m}$ belongs to $U$, then the property holds for $x$.

Lemma 1.5. Assume that $k$ is infinite. Any sequence which consists of $\alpha_{1}$ general elements in $J_{1}, \ldots, \alpha_{s}$ elements in $J_{s}$ forms an $\left(\alpha_{1}, \ldots, \alpha_{s}\right)$-superficial sequence for the ideals $J_{1}, \ldots, J_{s}$.

Proof. Let $x_{1}, \ldots, x_{m}$ be a sequence of such general elements, $m=\alpha_{1}+\cdots+\alpha_{s}$. Assume that $x_{i} \in J_{\varepsilon_{i}}$. Since $x_{i}$ is a general element of $J_{\varepsilon_{i}}$, we have $x_{i}^{*} \notin P$ for any associated prime $P$ of $\left(x_{1}^{*}, \ldots, x_{i-1}^{*}\right)$ with $P \nsupseteq J_{\varepsilon_{i}} / I J_{1} \ldots J_{\varepsilon_{i}-1} J_{\varepsilon_{i}}^{2} J_{\varepsilon_{i}+1} \ldots J_{s}$. Since $S_{+}$ is contained in the ideal generated by the elements of $J_{\varepsilon_{i}} / I J_{1} \ldots J_{\varepsilon_{i}-1} J_{\varepsilon_{i}}^{2} J_{\varepsilon_{i}+1} \ldots J_{s}$, this implies $x_{i}^{*} \notin P$ for any associated prime $P$ of $\left(x_{1}^{*}, \ldots, x_{i-1}^{*}\right)$ with $P \nsupseteq S_{+}$. Hence $x_{1}^{*}, \ldots, x_{m}^{*}$ is a filter-regular sequence in $S$.

Corollary 1.6. Assume that the local ring A has infinite residue field. Let $Q$ be an ideal generated by $\alpha_{1}$ general elements in $J_{1}, \ldots, \alpha_{n}$ elements in $J_{n}$. Then $e_{\alpha}\left(I \mid J_{1}, \ldots, J_{n}\right)>0$ if and only if $\operatorname{dim} A /\left(Q: J^{\infty}\right)=\alpha_{0}+1$. In this case,

$$
e_{\alpha}\left(I \mid J_{1}, \ldots, J_{n}\right)=e\left(I, A /\left(Q: J^{\infty}\right)\right) .
$$

Now we shall see that the characterization of mixed multiplicities of $\mathfrak{m}$-primary ideals given in [Te1] is a special case of Corollary 1.6.

Corollary 1.7 ([Te1, Ch. 0, Proposition 2.1]). Assume that the local ring $A$ has infinite residue field. Let $I, J_{1}, \ldots, J_{s}$ be $\mathfrak{m}$-primary ideals. Let $\alpha=\left(\alpha_{0}, \alpha_{1}, \ldots, \alpha_{s}\right)$ be any sequence of non-negative integers with $|\alpha|=\operatorname{dim} A-1$. Let $P$ be an ideal 
of $A$ generated by $\alpha_{0}+1$ general elements in $I, \alpha_{1}$ general elements in $J_{1}, \ldots, \alpha_{s}$ elements in $J_{s}$. Then

$$
e_{\alpha}\left(I \mid J_{1}, \ldots, J_{s}\right)=e(P, A) .
$$

Proof. Let $Q$ be the subideal of $P$ generated by $\alpha_{1}$ general elements in $J_{1}, \ldots, \alpha_{s}$ elements in $J_{s}$. By Lemma 1.5, these elements form a superficial sequence of the ideals $J_{1}, \ldots, J_{s}$. Since $J_{1}, \ldots, J_{s}$ are $\mathfrak{m}$-primary ideals, $Q$ is generated by a subsystem of parameters of $A$ and $J$ is an $\mathfrak{m}$-primary ideal. Therefore,

$$
\operatorname{dim} A /\left(Q: J^{\infty}\right)=\operatorname{dim} A / Q=\operatorname{dim} A-\left(\alpha_{1}+\cdots+\alpha_{s}\right)=\alpha_{0}+1 .
$$

By Theorem 1.4 and the above equation, we get

$$
e_{\alpha}\left(I \mid J_{1}, \ldots, J_{s}\right)=e\left(I, A /\left(Q: J^{\infty}\right)\right)=e(I, A / Q) .
$$

But $e(I, A / Q)=e(P, A / Q)$ because $P$ generates a minimal reduction of $I$ in $A / Q$. So we can conclude that

$$
e_{\alpha}\left(I \mid J_{1}, \ldots, J_{s}\right)=e(P, A / Q)=e(P, A) .
$$

Using Corollary 1.6 we obtain interesting properties of mixed multiplicities.

Corollary 1.8. Let $\alpha=\left(\alpha_{0}, \alpha_{1}, \ldots, \alpha_{s}\right)$ be any sequence of non-negative integers with $|\alpha|=d-1$. Assume that $e_{\alpha}\left(I \mid J_{1}, \ldots, J_{n}\right)>0$. Then

(a) $e_{\alpha}\left(I^{\prime} \mid J_{1}, \ldots, J_{n}\right)>0$ for any $\mathfrak{m}$-primary ideal $I^{\prime}$,

(b) $e_{\beta}\left(I \mid J_{1}, \ldots, J_{n}\right)>0$ for all $\beta=\left(\beta_{0}, \ldots, \beta_{n}\right)$ with $|\beta|=d-1$ and $\beta_{i} \leq \alpha_{i}$, $i=1, \ldots, n$.

Proof. Without loss of generality, we may assume that the residue field of $A$ is infinite. Let $Q$ be an ideal generated by $\alpha_{1}$ general elements in $J_{1}, \ldots, \alpha_{n}$ elements in $J_{n}$.

(a) By Corollary 1.6 the assumption implies $\operatorname{dim} A /\left(Q: J^{\infty}\right)=\alpha_{0}+1$. Since this condition does not depend on $I$, we also have $e_{\alpha}\left(I^{\prime} \mid J_{1}, \ldots, J_{n}\right)>0$.

(b) Let $Q^{\prime}$ denote the subideal of $Q$ generated by $\beta_{i}$ general elements in $J_{i}$, $i=1, \ldots, n$. Put $A^{*}=A / Q^{\prime}, I^{*}=I A^{*}$ and $J_{i}=J_{i} A^{*}$. Let $R^{*}=R\left(I^{*} \mid J_{1}^{*}, \ldots, J_{n}^{*}\right)$. By Lemma 1.3 we have

$$
P_{R^{*}}(u)=\Delta^{\left(0, \beta_{1}, \ldots, \beta_{n}\right)} P_{R}(u) .
$$

From this it follows that

$$
e_{\left(\alpha_{0}, \alpha_{1}-\beta_{1}, \ldots, \alpha_{n}-\beta_{n}\right)}\left(I^{*} \mid J_{1}^{*}, \ldots, J_{n}^{*}\right)=e_{\alpha}\left(I \mid J_{1}, \ldots, J_{n}\right)>0 .
$$

Hence $\operatorname{deg} P_{R^{*}}(u)=(d-1)-\left(\beta_{1}+\cdots+\beta_{n}\right)$. By Theorem 1.2(a), this implies

$$
\operatorname{dim} A /\left(Q^{\prime}: J^{\infty}\right)=\operatorname{deg} P_{R^{*}}(u)+1=\beta_{0}+1 .
$$

Therefore, $e_{\beta}\left(I \mid J_{1}, \ldots, J_{n}\right)>0$ by Corollary 1.6

\section{Mixed VOlumes AND TORIC RINGS}

The aim of this section is to interpret mixed volumes as mixed multiplicities.

Usually, a mixed volume is defined for a collection of $n$ convex polytopes in $\mathbb{R}^{n}$ (see e.g. [CLO]). But it is obvious that it may also be defined for any collection of 
convex polytopes in $\mathbb{R}^{n}$ as follows. Let $Q_{1}, \ldots, Q_{r}$ be convex polytopes in $\mathbb{R}^{n}$ with $\operatorname{dim}\left(Q_{1}+\cdots+Q_{r}\right) \leq r$. We call the value

$$
M V_{r}\left(Q_{1}, \ldots, Q_{r}\right):=\sum_{h=1}^{r} \sum_{1 \leq i_{1}<\ldots<i_{h} \leq r}(-1)^{r-h} V_{r}\left(Q_{i_{1}}+\cdots+Q_{i_{h}}\right)
$$

the mixed volume of $Q_{1}, \ldots, Q_{r}$. Here $V_{r}$ denotes the $r$-dimensional Euclidean volume.

Let $\mathbf{Q}=\left(Q_{1}, \ldots, Q_{s}\right)$ be a sequence of convex polytopes in $\mathbb{R}^{n}$. Let $\lambda=$ $\left(\lambda_{1}, \ldots, \lambda_{s}\right)$ be any sequence of non-negative integers. We denote by $\lambda \mathbf{Q}$ the Minkowski sum $\lambda_{1} Q_{1}+\cdots+\lambda_{s} Q_{s}$ and by $\mathbf{Q}_{\lambda}$ the multiset of $\lambda_{1}$ polytopes $Q_{1}, \ldots, \lambda_{s}$ polytopes $Q_{s}$. Minkowski showed that the volume of the polytope $\lambda \mathbf{Q}$ is a homogeneous polynomial in $\lambda$ whose coefficients are mixed volumes up to constants (see e.g. CLO, Ch. 7, Proposition 4.9]).

Proposition 2.1 (Minkowski formula). Let $r=\operatorname{dim}\left(Q_{1}+\cdots+Q_{s}\right)$. Then

$$
V_{r}(\lambda \mathbf{Q})=\sum_{\alpha \in \mathbb{N}^{s},|\alpha|=r} \frac{1}{\alpha !} M V_{r}\left(\mathbf{Q}_{\alpha}\right) \lambda^{\alpha} .
$$

We will use the Minkowski formula to establish the relationship between mixed volumes and mixed multiplicities. For that we need to work with graded toric rings.

Let $A=k\left[x_{1}, \ldots, x_{n}\right]$ be a polynomial ring over a field $k$. Let $M$ be a finite set of monomials in $A$. The subalgebra $k[M]$ of $A$ generated by the monomials of $M$ is called the toric ring (or affine semigroup ring) of $M$. We associate with every monomial $x_{1}^{a_{1}} \ldots x_{n}^{a_{n}} \in A$ the lattice point $a=\left(a_{1}, \ldots, a_{n}\right) \in \mathbb{N}^{n}$. Many ring-theoretic properties of $k[M]$ can be described by means of the lattice points of $M$ (see e.g. [BH, Section 6] or [Sta, Chap. I]). For instance,

$$
\operatorname{dim} k[M]=\operatorname{rank} \mathbb{Z}(M),
$$

where $\mathbb{Z}(M)$ denotes the subgroup of $\mathbb{Z}^{n}$ generated by the lattice points of $M$.

Assume furthermore that the lattice points of $M$ lie on an affine hyperlane of $\mathbb{R}^{n}$. This is for example the case when $M$ consists of monomials of the same degree. Then $k[M]$ has a natural $\mathbb{N}$-graded structure. The multiplicity $e(k[M])$ can be expressed in terms of the lattice points of $M$ as follows.

Let $Q_{M}$ denote the convex hull of the lattice points of $M$ in $\mathbb{R}^{n}$. Then $Q_{M}$ is a convex polytope with

$$
\operatorname{dim} Q_{M}=\operatorname{rank} \mathbb{Z}(M)-1 .
$$

Proposition 2.2. Let $r=\operatorname{rank} \mathbb{Z}(M)-1$. Let $E$ be any subset of $M$ such that its lattice points form a basis of $\mathbb{Z}(M)$. Then

$$
e(k[M])=\frac{V_{r}\left(Q_{M}\right)}{V_{r}\left(Q_{E}\right)} .
$$

This multiplicity formula is a consequence of Ehrhart's theory for the number of lattice points in lattice polytopes (see e.g. [BH, Theorem 6.3.12] or [Sta, Chap. I, Theorem 10.3]). The number $V_{r}\left(Q_{M}\right) / V_{r}\left(Q_{E}\right)$ is often called the normalized volume of the polytope $Q_{M}$ with respect to the lattice $\mathbb{Z}(M)$.

In the following we will be concerned with products of finite sets of monomials, which is the counterpart of Minkowski sums of convex polytopes.

Let $M_{1}, \ldots, M_{s}$ be sets of monomials in $A$ such that each $M_{i}$ consists of monomials of the same degree. For any sequence $\lambda=\left(\lambda_{1}, \ldots, \lambda_{s}\right)$ of positive integers we denote 
by $M^{\lambda}$ the set of all products of $\lambda_{1}$ monomials of $M_{1}, \ldots, \lambda_{s}$ monomials of $M_{s}$. Using the above propositions we can express the multiplicity of the toric ring $k\left[M^{\lambda}\right]$ in terms of mixed volumes.

Corollary 2.3. Let $r=\operatorname{rank} \mathbb{Z}\left(M^{(1, \ldots, 1)}\right)-1$. Let $E$ be any subset of $M^{(1, \ldots, 1)}$ such that its lattice points form a basis of $\mathbb{Z}\left(M^{(1, \ldots, 1)}\right)$. Let $\mathbf{Q}$ be the sequence of polytopes $Q_{M_{1}}, \ldots, Q_{M_{s}}$. Then

$$
e\left(k\left[M^{\lambda}\right]\right)=\frac{1}{V_{r}\left(Q_{E}\right)} \sum_{\alpha \in \mathbb{N}^{s},|\alpha|=r} \frac{1}{\alpha !} M V_{r}\left(\mathbf{Q}_{\alpha}\right) \lambda^{\alpha} .
$$

Proof. Every lattice vector of $M^{\lambda}$ is a sum of $\lambda_{1}$ lattice points of $M_{1}, \ldots, \lambda_{s}$ lattice points of $M_{s}$. Therefore,

$$
Q_{M^{\lambda}}=\lambda_{1} Q_{1}+\cdots+\lambda_{s} Q_{s}=\lambda \mathbf{Q}
$$

Since $\mathbb{Z}\left(M^{\lambda}\right)=\mathbb{Z}\left(M^{(1, \ldots, 1)}\right)$, we have $\operatorname{rank} \mathbb{Z}\left(M^{\lambda}\right)=r+1$. Using Proposition 2.2 we obtain

$$
e\left(k\left[M^{\lambda}\right]\right)=\frac{V_{r}(\lambda \mathbf{Q})}{V_{r}\left(Q_{E}\right)} .
$$

Hence the conclusion follows from Proposition 2.1.

This formula for the multiplicity of the toric rings $k\left[M^{\lambda}\right]$ resembles the formula for the multiplicity of diagonal subalgebras in Lemma 1.1. Therefore, if we can find a standard multigraded algebra such that the toric rings $k\left[M^{\lambda}\right]$ are its diagonal subalgebras, then a comparison of these formulas will imply a relationship between mixed volumes and mixed multiplicities.

Theorem 2.4. Let $A=k\left[x_{1}, \ldots, x_{n}\right]$ and $M_{0}, M_{1}, \ldots, M_{s}$ be a sequence of sets of monomials such that $M_{0}=\left\{x_{1}, \ldots, x_{n}\right\}$ and each $M_{i}$ consists of monomials of the same degree $d_{i}$ for $i=0,1, \ldots, s$. Let $\mathfrak{m}$ be the maximal graded ideal of $A$ and $J_{i}$ the ideal generated by the monomials of $M_{i}$. Let $R=R\left(\mathfrak{m} \mid J_{1}, \ldots, J_{s}\right)$ and let $\mathbf{Q}$ be the sequence of polytopes $Q_{M_{0}}, Q_{M_{1}}, \ldots, Q_{M_{s}}$. Then $\operatorname{deg} P_{R}(u)=n-1$ and for any $\alpha \in \mathbb{N}^{s+1}$ with $|\alpha|=n-1$,

$$
e_{\alpha}\left(\mathfrak{m} \mid J_{1}, \ldots, J_{s}\right)=\frac{M V_{n-1}\left(\mathbf{Q}_{\alpha}\right)}{\sqrt{n}} .
$$

Proof. Let $S$ denote the subalgebra of the polynomial ring $A\left[t_{0}, t_{1}, \ldots, t_{s}\right]$ generated by all monomials of the form $f_{i} t_{i}$ with $f_{i} \in M_{i}$. Then $S$ is a standard $\mathbb{N}^{s+1}$ graded algebra over $k$. We shall see that $R \cong S$ as $\mathbb{N}^{s+1}$-graded algebras. Let $u=\left(u_{0}, u_{1}, \ldots, u_{s}\right)$ be any sequence of non-negative integers. The vector space $R_{u}$ has a basis consisting of the monomials of $\mathfrak{m}^{u_{0}} J_{1}^{u_{1}} \ldots J_{s}^{u_{s}}$ which are not contained in $\mathfrak{m}^{u_{0}+1} J_{1}^{u_{1}} \ldots J_{s}^{u_{s}}$. Since each $J_{i}$ is generated by $M_{i}$ and since $M_{i}$ consists of monomials of the same degree, these monomials are of the form $f_{0} f_{1} \ldots f_{s}$, where each $f_{i}$ is a product of $u_{i}$ monomials of $M_{i}, i=0,1, \ldots, s$. By mapping the elements $f_{0} f_{1} \ldots f_{s} \in R_{u}$ to the elements $\left(f_{0} t_{0}^{u_{0}}\right)\left(f_{1} t_{1}^{u_{1}}\right) \ldots\left(f_{n} t_{n}^{u_{n}}\right) \in S_{u}$ we obtain an $\mathbb{N}^{s+1}$ graded isomorphism of $R$ and $S$.

Let $\lambda=\left(\lambda_{0}, \lambda_{1}, \ldots, \lambda_{s}\right)$ be any sequence of $s+1$ positive integers. The above isomorphism induces an $\mathbb{N}$-graded isomorphism of diagonal subalgebras $R^{\lambda} \cong S^{\lambda}$. Let $M^{\lambda}$ denote the set of all products of $\lambda_{0}$ monomials of $M_{0}, \ldots, \lambda_{s}$ monomials in $M_{s}$. Then

$$
S^{\lambda} \cong k\left[M^{\lambda}\right]
$$


Let $f$ be a product of $s$ monomials of $M_{1}, \ldots, M_{s}$. Put $E=\left\{x_{1} f, \ldots, x_{n} f\right\} \subseteq$ $M^{(1, \ldots, 1)}$. Then $\mathbb{Z}(E)$ contains all lattice points of the form $e_{i}-e_{j}$, where $e_{1}, \ldots, e_{n}$ denote the basic vectors of $\mathbb{R}^{n}$. Therefore, $\mathbb{Z}(E)$ contains all lattice points of the hyperplane $x_{1}+\cdots+x_{n}=\operatorname{deg} f+1$. Since all monomials of $M^{(1, \ldots, 1)}$ have the degree $\operatorname{deg} f+1$, the lattice points of $E$ form a basis for $\mathbb{Z}\left(M^{(1, \ldots, 1)}\right)$. Hence $\operatorname{rank} \mathbb{Z}\left(M^{(1, \ldots, 1)}\right)=n$. Since $Q_{E}$ is congruent to the convex polytope spanned by the points $e_{i}$,

$$
V_{n-1}\left(Q_{E}\right)=\frac{\sqrt{n}}{(n-1) !}
$$

Applying Corollary 2.3 we get

$$
e\left(S^{\lambda}\right)=\frac{(n-1) !}{\sqrt{n}} \sum_{\alpha \in \mathbb{N}^{s+1},|\alpha|=n-1} \frac{1}{\alpha !} M V_{n-1}\left(\mathbf{Q}_{\alpha}\right) \lambda^{\alpha} .
$$

On the other hand, since $\operatorname{dim} S^{\lambda}=\operatorname{rank} \mathbb{Z}\left(M^{\lambda}\right)=n$, using Lemma 1.1 we get $\operatorname{deg} P_{S}(u)=n-1$ and

$$
e\left(S^{\lambda}\right)=(n-1) ! \sum_{\alpha \in \mathbb{N}^{s+1},|\alpha|=n-1} \frac{1}{\alpha !} e_{\alpha}(S) \lambda^{\alpha} .
$$

Since the above two formulas for $e\left(S^{\lambda}\right)$ hold for all sequences $\lambda$ of positive integers, we can conclude that their corresponding terms are equal. This means

$$
e_{\alpha}(S)=\frac{M V_{n-1}\left(\mathbf{Q}_{\alpha}\right)}{\sqrt{n}}
$$

for any $\alpha \in \mathbb{N}^{s+1}$ with $|\alpha|=n-1$.

It is now easy to interpret mixed volumes as mixed multiplicities of ideals.

Corollary 2.5. Let $Q_{1}, \ldots, Q_{n}$ be an arbitrary collection of lattice convex polytopes in $\mathbb{R}^{n}$. Let $A=k\left[x_{0}, x_{1}, \ldots, x_{n}\right]$ and let $\mathfrak{m}$ be the maximal graded ideal of $A$. Let $M_{i}$ be any set of monomials of the same degree in $A$ such that $Q_{i}$ is the convex hull of the lattice points of their dehomogenized monomials in $k\left[x_{1}, \ldots, x_{n}\right]$. Let $J_{i}$ be the ideal of A generated by the monomials of $M_{i}$. Then

$$
M V_{n}\left(Q_{1}, \ldots, Q_{n}\right)=e_{(0,1, \ldots, 1)}\left(\mathfrak{m} \mid J_{1}, \ldots, J_{n}\right) .
$$

Proof. By definition, the projection of the lattice point of a monomial on the hyperplane $x_{0}=0$ is the lattice point of its dehomogenized monomial. Therefore, the convex hull $Q_{M_{i}}$ of the lattice points of $M_{i}$ is the projection of the polytope $Q_{i}$ on the hyperplane $x_{0}=0$. As a consequence, the volume $V_{n}\left(Q_{M_{i}}\right)$ is proportional to $V_{n}\left(Q_{i}\right)$. This proportion can be computed as the volume of the convex hull $Q_{E}$ of the basic vectors $e_{0}, \ldots, e_{n}$ of $\mathbb{R}^{n+1}$. Since $V_{n}\left(Q_{E}\right)=\sqrt{n+1}$, we obtain

$$
V_{n}\left(Q_{i}\right)=\frac{V_{n}\left(Q_{M_{i}}\right)}{\sqrt{n+1}}
$$

From this it follows that the corresponding mixed volumes are also proportional:

$$
M V_{n}\left(Q_{1}, \ldots, Q_{n}\right)=\frac{M V_{n}\left(Q_{M_{1}}, \ldots, Q_{M_{n}}\right)}{\sqrt{n+1}}
$$


On the other hand, applying Theorem 2.4 to the sequence $M_{0}, M_{1}, \ldots, M_{n}$ of monomials in $n+1$ variables we obtain

$$
e_{(0,1, \ldots, 1)}\left(\mathfrak{m} \mid J_{1}, \ldots, J_{n}\right)=\frac{M V_{n}\left(Q_{M_{1}}, \ldots, Q_{M_{n}}\right)}{\sqrt{n+1}} .
$$

Therefore, we can conclude that $M V_{n}\left(Q_{1}, \ldots, Q_{n}\right)=e_{(0,1, \ldots, 1)}\left(\mathfrak{m} \mid J_{1}, \ldots, J_{n}\right)$.

An immediate consequence of the interpretation of mixed volumes as mixed multiplicities is the non-trivial fact that mixed volumes are always non-negative numbers. In fact, we can reprove the following result given in [Fu2, p. 117].

Corollary 2.6. Let $P_{1}, \ldots, P_{n}$ and $Q_{1}, \ldots, Q_{n}$ be two sequences of convex lattice polytopes in $\mathbb{R}^{n}$ with $P_{i} \supseteq Q_{i}$. Then

$$
M V_{n}\left(P_{1}, \ldots, P_{n}\right) \geq M V_{n}\left(Q_{1}, \ldots, Q_{n}\right) .
$$

Proof. By Corollary 2.5 we have

$$
\begin{aligned}
M V_{n}\left(P_{1}, \ldots, P_{n}\right) & =e_{(0,1, \ldots, 1)}\left(\mathfrak{m} \mid I_{1}, \ldots, I_{n}\right), \\
M V_{n}\left(Q_{1}, \ldots, Q_{n}\right) & =e_{(0,1, \ldots, 1)}\left(\mathfrak{m} \mid J_{1}, \ldots, J_{n}\right),
\end{aligned}
$$

where $I_{i}$ and $J_{i}$ are ideals generated by monomial ideals with the same degree and $I_{i} \supseteq J_{i}$. Note that the vector space $\mathfrak{m}^{u_{0}} I_{1}^{u_{1}} \ldots I_{n}^{u_{n}} / \mathfrak{m}^{u_{0}+1} I_{1}^{u_{1}} \ldots I_{n}^{u_{n}}$ contains the vector space $\mathfrak{m}^{u_{0}} J_{1}^{u_{1}} \ldots J_{n}^{u_{n}} / \mathfrak{m}^{u_{0}+1} J_{1}^{u_{1}} \ldots J_{n}^{u_{n}}$ for all $u=\left(u_{0}, u_{1}, \ldots, u_{n}\right) \in \mathbb{N}^{n+1}$. Then

$$
H_{R\left(\mathfrak{m} \mid I_{1}, \ldots, I_{n}\right)}(u) \geq H_{R\left(\mathfrak{m} \mid J_{1}, \ldots, J_{n}\right)}(u) .
$$

Since $e_{(0,1, \ldots, 1)}\left(\mathfrak{m} \mid I_{1}, \ldots, I_{n}\right)$ and $e_{(0,1, \ldots, 1)}\left(\mathfrak{m} \mid J_{1}, \ldots, J_{n}\right)$ are the coefficients of one of the leading terms of the corresponding Hilbert polynomials, we obtain

$$
e_{(0,1, \ldots, 1)}\left(\mathfrak{m} \mid I_{1}, \ldots, I_{n}\right) \geq e_{(0,1, \ldots, 1)}\left(\mathfrak{m} \mid J_{1}, \ldots, J_{n}\right),
$$

which implies the conclusion.

Remark. Relations among mixed volumes of lattice polytopes always hold for arbitrary convex polytopes by approximating them with rational convex polytopes and then using finer lattices [Te3].

Now we come to the famous Alexandroff-Fenchel inequality between mixed volumes:

$$
M V_{n}\left(Q_{1}, \ldots, Q_{n}\right)^{2} \geq M V_{n}\left(Q_{1}, Q_{1}, Q_{3}, \ldots, Q_{n}\right) M V_{n}\left(Q_{2}, Q_{2}, Q_{3}, \ldots, Q_{n}\right) .
$$

Khovanski $\mathrm{Kh}$ ] and Teissier [Te3] used the Hodge index theorem in intersection theory to prove this inequality. This leads us to believe that a similar inequality should hold between mixed multiplicities.

Question 2.7. Let $(A, \mathfrak{m})$ be a local (or standard graded) $\operatorname{ring}$ with $\operatorname{dim} A=$ $n+1 \geq 3$. Let $I$ be an $\mathfrak{m}$-primary ideal and $J_{1}, \ldots, J_{n}$ ideals of height $n$. Put $\alpha=(0,1, \ldots, 1)$. Is it true that

$$
e_{\alpha}\left(I \mid J_{1}, \ldots, J_{n}\right)^{2} \geq e_{\alpha}\left(I \mid J_{1}, J_{1}, J_{3}, \ldots, J_{n}\right) e_{\alpha}\left(I \mid J_{2}, J_{2}, J_{3}, \ldots, J_{n}\right) ?
$$

Using Theorem 1.4 we can reduce this theorem to the case $\operatorname{dim} A=3$. In this case, we have to prove the simpler formula:

$$
e_{(0,1,1)}\left(I \mid J_{1}, J_{2}\right)^{2} \geq e_{(0,1,1)}\left(I \mid J_{1}, J_{1}\right) e_{(0,1,1)}\left(I \mid J_{2}, J_{2}\right) .
$$

Unfortunately, we were unable to give an answer to the above question. The difficulty can be seen from the following observation. 
Remark. The above inequality does not hold if $J_{1}, \ldots, J_{n}$ are $\mathfrak{m}$-primary ideals. In this case, we can even show that the inverse inequality holds, namely,

$$
e_{\alpha}\left(I \mid J_{1}, \ldots, J_{n}\right)^{2} \leq e_{\alpha}\left(I \mid J_{1}, J_{1}, J_{3}, \ldots, J_{n}\right) e_{\alpha}\left(I \mid J_{2}, J_{2}, J_{3}, \ldots, J_{n}\right)
$$

where $\alpha=(0,1,1, \ldots, 1)$. Using Corollary 1.7 we can translate it to the inequality

$$
e_{(1,1)}\left(J_{1} \mid J_{2}\right)^{2} \leq e\left(J_{1}, A\right) e\left(J_{2}, A\right)
$$

for a two-dimensional ring $A$. This inequality was proved first by Teissier Te2] for reduced Cohen-Macaulay rings over an algebraically closed field of characteristic zero and then by Rees and Sharp [RS] in general.

It is known that computing mixed volumes is a hard enumerative problem (see EC], HS1, HS2 for algorithms and software for doing these computations). Instead of that we can now compute mixed multiplicities of the associated graded ring of the multigraded Rees algebra $A\left[J_{1} t_{1}, \ldots, J_{n} t_{n}\right]$ with respect to the ideal $\mathfrak{m}$. By Corollary 1.6, these mixed multiplicities can be interpreted as Samuel multiplicities. The computation of these multiplicities can be carried out by computer algebra systems such as Cocoa, Macaulay 2 and Singular.

\section{Bernstein's THEOREM}

Let $k\left[x_{1}^{ \pm 1}, \ldots, x_{n}^{ \pm 1}\right]$ be a Laurent polynomial ring over a field $k$. For any Laurent polynomial

$$
f=\sum_{a \in \mathbb{Z}^{n}} c_{a} x^{a}\left(c_{a} \in k\right)
$$

we will denote by $M(f)$ the set of monomials $x^{a}$ with $c_{a} \neq 0$. Let $Q_{f}$ denote the convex hull of the lattice points $a$ with $c_{a} \neq 0$ in $\mathbb{R}^{n}$, i.e. $Q_{f}=Q_{M(f)}$. One calls $Q_{f}$ the Newton polytope of $f$.

Bernstein's theorem says that the mixed volume of the associated Newton polytopes of $n$ Laurent polynomials is a sharp bound for the number of common zeros in the torus $\left(\mathbb{C}^{*}\right)^{n}[\mathrm{Be}$, Theorem A]. Here we will prove Bernstein's theorem by purely algebraic means for any algebraically closed field $k$.

Theorem 3.1. Let $k$ be an algebraically closed field. Let $f_{1}, \ldots, f_{n}$ be Laurent polynomials in $k\left[x_{1}^{ \pm 1}, \ldots, x_{n}^{ \pm 1}\right]$ with finitely many common zeros in $\left(k^{*}\right)^{n}$. Then the number of common zeros of $f_{1}, \ldots, f_{n}$ in $\left(k^{*}\right)^{n}$ is bounded above by $M V_{n}\left(Q_{f_{1}}, \ldots, Q_{f_{n}}\right)$. Moreover, this bound is attained for a generic choice of coefficients in $f_{1}, \ldots, f_{n}$ if $k$ has characteristic zero.

Here, a generic choice of coefficients in $f_{1}, \ldots, f_{n}$ means that the supporting monomials of $f_{1}, \ldots, f_{n}$ remain the same while their coefficients vary in a non-empty open parameter space.

Now we are going to give a homogeneous version of Bernstein's theorem.

Let $f^{h}$ denote the homogenization of a Laurent polynomial $f$ in $k\left[x_{0}^{ \pm 1}, x_{1}^{ \pm 1}, \ldots\right.$, $\left.x_{n}^{ \pm 1}\right]$. Then $Q_{f^{h}}$ is a polytope in $\mathbb{R}^{n+1}$. Its projection to the hyperplane $x_{0}=0$ is a polytope canonically identified with $Q_{f}$. We have $V_{n}\left(Q_{f^{h}}\right)=\sqrt{n+1} V_{n}\left(Q_{f}\right)$. Hence

$$
M V_{n}\left(Q_{f_{1}^{h}}, \ldots, Q_{f_{n}^{h}}\right)=\sqrt{n+1} M V_{n}\left(Q_{f_{1}}, \ldots, Q_{f_{n}}\right) .
$$

It is also obvious that the number of common zeros of $f_{1}, \ldots, f_{n}$ in $\left(k^{*}\right)^{n}$ is equal to the number of common zeros of $f_{1}^{h}, \ldots, f_{n}^{h}$ in $\mathbb{P}_{k^{*}}^{n}$, where $\mathbb{P}_{k^{*}}^{n}$ denotes the set of all 
points of the projective space $\mathbb{P}_{k}^{n}$ with non-zero components. Thus, Theorem 3.1 can be translated as follows.

Theorem 3.2. Let $k$ be an algebraically closed field. Let $g_{1}, \ldots, g_{n}$ be homogeneous Laurent polynomials in $k\left[x_{0}^{ \pm 1}, x_{1}^{ \pm 1}, \ldots, x_{n}^{ \pm 1}\right]$ with finitely many common zeros in $\mathbb{P}_{k^{*}}^{n}$. Then

$$
\left|\left\{\alpha \in \mathbb{P}_{k^{*}}^{n} \mid g_{i}(\alpha)=0, i=1,2, \ldots, n\right\}\right| \leq \frac{M V_{n}\left(Q_{g_{1}}, \ldots, Q_{g_{n}}\right)}{\sqrt{n+1}} .
$$

Moreover, this bound is attained for a generic choice of coefficients in $g_{1}, \ldots, g_{n}$ if $k$ has characteristic zero.

We may reduce the above theorems to the case of polynomials. In fact, if we multiply the given Laurent polynomials with an appropriate monomial, then we will obtain a new system of polynomials. Obviously, the new polynomials in $\left(k^{*}\right)^{n}$ or in $\mathbb{P}_{k^{*}}^{n}$ have the same common zeros. Since their Newton polytopes are translations of the old ones, their mixed volumes do not change, too.

Now assume that $g_{1}, \ldots, g_{n}$ are homogeneous polynomials in $A=k\left[x_{0}, x_{1}, \ldots, x_{n}\right]$. Let $M_{i}$ be the set of monomials occuring in $g_{i}$. Let $\mathfrak{m}$ be the maximal graded ideal of $A$ and $J_{i}$ the ideals of $A$ generated by $M_{i}$. Put

$$
R=R\left(\mathfrak{m} \mid J_{1}, \ldots, J_{n}\right) .
$$

We know by Theorem 2.4 that $\operatorname{deg} P_{R}(u)=n+1$ and

$$
e_{(0,1, \ldots, 1)}(R)=\frac{M V_{n}\left(Q_{g_{1}}, \ldots, Q_{g_{n}}\right)}{\sqrt{n+1}} .
$$

Therefore, Theorem 3.2 follows from the following result.

Theorem 3.3. Let $k$ be an algebraically closed field. Let $g_{1}, \ldots, g_{n}$ be homogeneous polynomials in $k\left[x_{0}, x_{1}, \ldots, x_{n}\right]$ with finitely many common zeros in $\mathbb{P}_{k^{*}}^{n}$. Then

$$
\left|\left\{\alpha \in \mathbb{P}_{k^{*}}^{n} \mid g_{i}(\alpha)=0, i=1,2, \ldots, n\right\}\right| \leq e_{(0,1, \ldots, 1)}(R) .
$$

Moreover, this bound is attained for a generic choice of coefficients in $g_{1}, \ldots, g_{n}$ if $k$ has characteristic zero.

Proof. Let $Q$ be the ideal $\left(g_{1}, \ldots, g_{n}\right)$. Then there is a one-to-one correspondence between common zeros of $g_{1}, \ldots, g_{n}$ in $\mathbb{P}_{k^{*}}^{n}$ and the one-dimensional homogeneous primes of $A$ which contain $Q:\left(x_{0} \ldots x_{n}\right)^{\infty}$. As a consequence, the assumption on $g_{1}, \ldots, g_{n}$ implies that $Q:\left(x_{0} \ldots x_{n}\right)^{\infty}$ is a one-dimensional ideal. Therefore, the number of common zeros of $g_{1}, \ldots, g_{n}$ in $\mathbb{P}_{k^{*}}^{n}$ is equal to the number of minimal associated prime ideals of $Q:\left(x_{0} \ldots x_{n}\right)^{\infty}$ which is bounded above by the multiplicity $e\left(A /\left(Q:\left(x_{0} \ldots x_{n}\right)^{\infty}\right)\right)$ in view of the associativity formula for multiplicities. By the principle of conservation of number (see e.g. Fulton [Fu1, Section 10.2]), we only need to show that for a generic choice of the coefficients of $g_{1}, \ldots, g_{n}, Q:\left(x_{0} \ldots x_{n}\right)^{\infty}$ there is a radical ideal with

$$
e\left(A /\left(Q:\left(x_{0} \ldots x_{n}\right)^{\infty}\right)\right)=e_{(0,1, \ldots, 1)}(R) .
$$

Let $J:=J_{1} \ldots J_{n}$. We may multiply $g_{1}, \ldots, g_{n}$ with $x_{0} \ldots x_{n}$ to obtain a new system of equations with $J \subseteq\left(x_{0} \ldots x_{n}\right)$. Since $\left(x_{0} \ldots x_{n}\right)^{m} \in J$ for $m \gg 0$,

$$
Q:\left(x_{0} \ldots x_{n}\right)^{\infty}=Q: J^{\infty} .
$$

By Corollary [1.6 we have for a generic choice of the coefficients of $g_{1}, \ldots, g_{n}$,

$$
e\left(A /\left(Q: J^{\infty}\right)\right)=e_{(0,1, \ldots, 1)}(R)
$$


Thus, the number of common zeros of $g_{1}, \ldots, g_{n}$ in $\mathbb{P}_{k^{*}}^{n}$ is bounded by the mixed multiplicity $e_{(0,1, \ldots, 1)}(R)$. It remains to show that $Q: J^{\infty}$ is a radical ideal for a generic choice of the coefficients of $g_{1}, \ldots, g_{n}$ if $k$ has characteristic zero. But this follows from Bertini theorem [Fl, Satz 5.4(e)].

Finally, we would like to remark that the last statement of the above theorems does not hold if the ground field has positive characteristic.

Example. Let $k$ be an algebraically closed field with $\operatorname{char}(k)=p$. Let $f(x)=$ $a x^{p}+b$ be a polynomial in one variable, $a, b \in k$. For $a, b \neq 0$ we choose $c \in k$ such that $c^{p}=b / a$. Then $f(x)=a(x+c)^{p}$ has only one zero in $k^{*}$, whereas the Newton polygon of $f$ has volume $p$.

\section{ACKNOWLEDGMENTS}

The work on this paper began while the second author visited the Institute of Mathematics, Hanoi in August 2002 under the China-India-Vietnam Network on Commutative Algebra and Algebraic Geometry established by the Abdus Salam International Centre for Theoretical Physics (ICTP), Trieste, Italy. He thanks both institutions for their support.

\section{REFERENCES}

[Ba] P. B. Bhattacharya, The Hilbert function of two ideals, Proc. Cambridge Phil. Soc. 53 (1957), 568-575. MR0089835(19:727b)

[Be] D. N. Bernstein, The number of roots of a system of equations (Russian), Funkcional. Anal. i Priložen. 9 (1975), no. 3, 1-4. MR0435072 (55:8034)

[BF] T. Bonnesen and W. Fenchel, Theorie der konvexen Körper, Chelsea, New York, 1971. MR0372748(51:8954)

[BH] W. Bruns and J. Herzog, Cohen-Macaulay Rings, Revised Edition, Cambridge Studies in Advanced Mathematics, 39. Cambridge University Press, Cambridge, 1998. MR:1251956 (95h:13020)

[CHTV] A. Conca, J. Herzog, N.V. Trung and G. Valla, Diagonal subalgebras of bigraded algebras and embeddings of blow-ups of projective spaces, American Journal of Math. 119 (1997), 859-901. MR.1465072 (99d:13001)

[CLO] D. Cox, J. Little and D. O'Shea, Using Algebraic Geometry, Springer, New York, 1998. MR:1639811 (99h:13033)

[EC] I. Emiris and J. Canny, Efficient incremental algorithms for the sparse resultant and the mixed volume, J. Symbolic Comput. 20 (1995), 117-149. MR.1374227 (96j:68098)

[Ew] G. Ewald, Combinatorial convexity and algebraic geometry, Springer, New York, 1996. MR.1418400 (97i:52012)

[Fl] H. Flenner, Die Sätze von Bertini für lokale Ringe, Math. Ann. 229 (1977), 97-111. MR0460317 (57:311)

[Fu1] W. Fulton, Intersection theory, Springer-Verlag, Berlin-Heidelberg, 1984. MR0732620 (85k:14004)

[Fu2] W. Fulton, Introduction to toric varieties, Annals of Mathematics Studies, 131, Princeton University Press, 1993. MR.1234037 (94g:14028)

[GKZ] I. M. Gelfand, M. Kapranov and A. Zelevinsky, Discriminants, Resultants and Multidimensional Determinants, Birkhäuser, Boston, 1994. MR1264417 (95e:14045)

[HS1] B. Huber and B. Sturmfels, A polyhedral method for solving sparse polynomial equations, Math. of Computation 64 (1995), 1541-1555. MR.1297471 (95m:65100)

[HS2] B. Huber and B. Sturmfels, Bernstein's theorem in affine spaces, Discrete. Comput. Geom. 19 (1997), 137-141. MR1424821 (98b:52014)

[KaMV] D. Katz, S. Mandal and J. Verma, Hilbert function of bigraded algebras, in: A. Simis, N. V. Trung and G. Valla (eds.), Commutative Algebra (ICTP, Trieste, 1992), 291-302, World Scientific, 1994. MR:1421092 (97h:13013) 
[KaV] D. Katz and J. Verma, Extended Rees algebras and mixed multiplicities, Math. Z. 202 (1989), 111-128. MR1007742 (90i:13024)

[Kh] A. G. Khovanski, Newton polytopes and toric varieties, Functional Anal. Appl. 11 (1977), 289-298. MR0476733 (57:16291)

$[\mathrm{Ku}] \quad$ A. G. Kuschnirenko, Newton polytopes and Bezout theorem, Functional Anal. Appl. 10 (1976), 233-235. MR0422272 (54:10263)

[R1] D. Rees, a-transforms of local rings and a theorem on multiplicities of ideals, Proc. Cambridge Philos. Soc. 57 (1961), 8-17. MR0118750 (22:9521)

[R2] D. Rees, Generalizations of reductions and mixed multiplicities, J. London Math. Soc. 29 (1984), 423-432. MR 0754926 (86e:13023)

[Ro] P. Roberts, Local Chern classes, multiplicities and perfect complexes, Mémoire Soc. Math. France 38 (1989), 145-161. MR.1044350 (91d:13025)

[RS] D. Rees and R. Y. Sharp, On a theorem of B. Teissier on mixed multiplicities of ideals in local rings, J. London Math. Soc. 18 (1978), 449-463. MR0518229 (80e:13009)

[Sta] R. P. Stanley, Combinatorics and Commutative Algebra, Birhäuser, Boston, 1986. MR0725505 (85b:05002)

[Stu] B. Sturmfels, Solving systems of polynomial equations, CBMS Regional Conference Series in Mathematics, No. 97, American Mathematical Society, 2002. MR:1925796 (2003i:13037)

[SV] J. Stückrad and W. Vogel, Buchsbaum rings and applications, VEB Deutscher Verlag der Wisssenschaften, Berlin, 1986. MR0881220 (88h:13011b)

[Sw] I. Swanson, Mixed multiplicities, joint reductions and quasi-unmixed local rings, J. London Math. Soc. 48 (1993), 1-14. MR1223888 (94d:13027)

[Te1] B. Teissier, Cycles évanescents, sections planes, et conditions de Whitney, Singularités à Cargèse 1972, Astèrisque 7-8 (1973), 285-362. MR 0374482 (51:10682)

[Te2] B. Teissier, Sur un inégalité à la Minkowski pour les multiplicités (Appendix to a paper by D. Eisenbud and H. I. Levine), Ann. of Math. 106 (1977), 38-44. MR0467800 $(57: 7651)$

[Te3] B. Teissier, Du théorème de l'index de Hodge aux inégalités isopérimétriques, C. R. Acad. Sci. Paris Ser. A-B 288 (1979), no. 4, A287-A289. MR0524795 (80k:14014)

[Tr1] N. V. Trung, The Castelnuovo regularity of the Rees algebra and the associated graded ring, Trans. Amer. Math. Soc. 350 (1998), 2813-2832. MR1473456 (98j:13006)

[Tr2] N. V. Trung, Positivity of mixed multiplicities, Math. Ann. 319 (2001), 33-63. MR:1812818 (2001m:13042)

[Va] G. Valla, Certain graded algebras are always Cohen-Macaulay, J. Algebra, 42 (1976), 537-548. MR0422249(54:10240)

[Vi] D. Q. Viet, Mixed multiplicities of arbitrary ideals in local rings, Comm. Algebra 28 (8) (2000), 3803-3821. MR.1767591 (2001f:13036)

[Ve1] J. K. Verma, Rees algebras and mixed multiplicities, Proc. Amer. Math. Soc. 104 (1988), 1036-1044. MR.0929432 (89d:13018)

[Ve2] J. K. Verma, Multigraded Rees algebras and mixed multiplicities, J. Pure Appl. Algebra 77 (1992), 219-228. MR1149023 (93e:13005)

[Wa] B. L. Van der Waerden, On Hilbert's function, series of composition of ideals and a generalization of the theorem of Bezout, Proc. K. Akad. Wet. Amsterdam 31 (1928), 749-770.

Institute of Mathematics, Viên ToÁn Hoc, 18 HoÀng Quôc Viêt, 10307 Hanoi, Vietnam

E-mail address: nvtrung@math.ac.vn

Department of Mathematics, Indian Institute of Technology Bombay, Mumbai, India 400076

E-mail address: jkv@math.iitb.ac.in 